\title{
Un aspect de l'implication de l'École dans la Grande Guerre : l'hommage aux « maîtres morts pour la France » dans l'académie de Poitiers (1914-1930)
}

An aspect of the involvement of schools during the Great War: an homage to the " maitres morts pour la France " in the local education authority of Poitiers

(1914-1930)

Hugues Marquis

\section{(2) OpenEdition}

Journals

Édition électronique

URL : http://journals.openedition.org/abpo/3067

DOI : $10.4000 / a b p o .3067$

ISBN : 978-2-7535-4275-4

ISSN : $2108-6443$

\section{Éditeur}

Presses universitaires de Rennes

\section{Édition imprimée}

Date de publication : 30 juin 2015

Pagination : 119-137

ISBN : 978-2-7535-4273-0

ISSN : 0399-0826

\section{Référence électronique}

Hugues Marquis, « Un aspect de l'implication de l'École dans la Grande Guerre : I'hommage aux " maîtres morts pour la France » dans l'académie de Poitiers (1914-1930) », Annales de Bretagne et des Pays de l'Ouest [En ligne], 122-2 | 2015, mis en ligne le 30 juin 2017, consulté le 19 avril 2019. URL : http://journals.openedition.org/abpo/3067 ; DOI : 10.4000/abpo.3067 


\title{
Un aspect de l'implication de l'École dans la Grande Guerre : l'hommage aux "maîtres morts pour la France " dans l'académie de Poitiers (1914-1930)
}

\author{
Hugues MARQUIS \\ docteur en histoire, professeur agrégé à l'ESPE - Université de Poitiers, \\ chercheur associé au CRIHAM (EA 4270 Poitiers-Limoges)
}

Au lendemain de la Grande Guerre, la quasi-totalité des 36000 communes françaises érigent des monuments qui rendent hommage à leurs habitants morts à la guerre. À côté de cela, on voit aussi se multiplier les monuments émanant de différentes professions et qualifiés de " corporatifs ${ }^{1}$ ". Les instituteurs, qui ont payé un lourd tribut à la guerre, sont honorés dans des monuments qui sont érigés dans l'enceinte des écoles normales dans la quasi-totalité des départements. Rappelons que, dans ce contexte mémoriel, le terme de monument désigne une " construction faite pour transmettre à la postérité la mémoire de quelque personnage illustre, ou de quelque événement considérable ${ }^{2}$ ". Les textes de l'époque se rapportant aux différentes formes d'hommage aux morts de la Grande Guerre utilisent tous le terme de " monument " pour désigner indifféremment les plaques, stèles ou ensembles monumentaux. Ils sont présents dans les huit départements qui composent alors l'académie de Poitiers ${ }^{3}$. Ces monuments ne sont que la forme la plus visible dans l'espace de l'hommage rendu par l'École à ses maîtres, hommage qui se manifeste aussi par d'autres supports comme les livres d'or, tableaux d'honneur et médailles, ou par des cérémonies commémoratives. C'est dès le début du conflit que le ministère

1. Sur cette diversité mémorielle, voir BECKER, Annette, Les monuments aux morts : mémoire de la Grande Guerre, Paris, Errance, 1988, et Prost, Antoine, "Les monuments aux morts ", dans NorA, Pierre, Les lieux de mémoire, t. I, La République, Paris, Gallimard, 1984.

2. LiTTRÉ, Émile, Dictionnaire de la langue française, tome troisième, Paris, Hachette, 1874 , p. 621.

3. Charente, Charente-Maritime, Deux-Sèvres, Indre, Indre-et-Loire, Vendée, Vienne, Haute-Vienne. 
de l'Instruction publique manifeste son intention de rendre hommage à la participation des instituteurs, en particulier à ceux qui tombent au combat. Cet hommage vise à la fois à souligner la participation du corps enseignant à la guerre et à en organiser la mémoire.

Il s'agit donc ici de s'interroger sur les modalités de l'hommage rendu aux enseignants du primaire morts durant la Première Guerre mondiale, d'en étudier les formes et les origines, afin de comprendre la spécificité de cette mémoire corporative. L'exemple de l'académie de Poitiers est intéressant parce que l'on y observe des manifestations précoces et diverses de cet hommage aux maîtres morts à la guerre.

\section{Le Livre d'or de l'Université}

La première forme d'hommage aux instituteurs engagés dans la guerre est celle du livre d'or, qui désigne, dans son sens actuel, une liste des personnes qui ont accompli une action mémorable dans un domaine déterminé 4 .

\section{Une mise en place dès les premiers mois de la guerre}

C'est une circulaire du ministre de l'Instruction publique Albert-Sarrault du 12 septembre 1914 qui demande aux recteurs d'académie d'ouvrir le Livre d'or de l'Université. Parmi les 25000 membres de l'enseignement public qui " combattent pour le salut de la France ", on retiendra " pieusement les noms de ceux qui accompliront des actions d'éclat ou qui verseront leur sang pour la Patrie ». Dans chaque département sera donc dressée par l'Inspection académique, à partir des documents officiels, la liste des membres de l'enseignement public, " qui auront mérité, pendant la campagne, une promotion de grade, une distinction militaire, une citation à l'ordre de l'armée " et « la liste de ceux qui seront tombés, morts ou blessés, sur le champ de bataille". Ce sont ces listes qui formeront un "véritable livre d'or de l'Université ${ }^{5}$ ".

La demande du ministre est rapidement mise en œuvre dans l'académie de Poitiers par une circulaire adressée par le recteur aux inspecteurs d'académie, le 23 septembre, circulaire qui reprend en substance celle du ministre ${ }^{6}$; dans les départements, les inspecteurs donnent des consignes pour recueillir les informations. L'inspecteur d'académie de la Charente demande, aux familles des instituteurs mobilisés et à leurs collègues de la même commune, de faire connaître "sans retard, les actions d'éclat, promotion de grade, distinction militaire, citation à l'ordre du jour, par lesquelles se seraient signalés les professeurs ou les instituteurs publics ",

\footnotetext{
4. Dictionnaire de l'Académie française, neuvième édition, en cours de rédaction.

5. Bulletin de l'Instruction primaire de la Vendée, octobre-novembre 1914.

6. Bulletin départemental de l'Instruction primaire (Charente), supplément au bulletin $n^{\circ} 263$.
} 
s'appuyant sur un formulaire type, à envoyer à l'Inspection académique départementale par l'intermédiaire de l'inspecteur primaire ${ }^{7}$.

En Charente, c'est le Bulletin départemental de juillet-août-septembre 1914 qui ouvre le "Livre d'or de l'Université " pour le département en publiant la première liste des tués et blessés au combat ${ }^{8}$. Deux instituteurs adjoints sont " morts au champ d'honneur " et 15 instituteurs ou instituteurs adjoints, ainsi que deux professeurs de collège et lycée, sont blessés. Le premier inscrit est Jacques Albert, instituteur adjoint à Chalais, soldat au $250^{\mathrm{e}}$ régiment d'infanterie tué d'un éclat d'obus à Tracy-le-Mont dans l'Oise le 27 septembre 1914.

Dans la Vienne, le Livre d'or est ouvert dans le numéro d'octobrenovembre 1914 du Bulletin départemental. Cinq noms de maîtres de l'enseignement primaire sont sur cette première page, auxquels s'ajoutent six blessés, dont un professeur de l'école normale de Poitiers et un élève-maître de cette école ${ }^{9}$. Pour les personnels du département de la Haute-Vienne, le Livre d'or n'est ouvert qu'en janvier $1915^{10}$, et pour le département de la Vendée, qu'en avril $1915^{11}$. C'est du moins la première mention des maîtres morts au combat dans les bulletins départementaux.

Le Livre d'or occupe toujours la première page du Bulletin départemental de l'instruction primaire ou la première page de la partie départementale de ce bulletin. Conformément aux instructions du recteur, les pages constituant le Livre d'or de l'Université renseignent pour chaque " tué à l'ennemi " le nom, la fonction dans l'Éducation nationale (instituteur, professeur, instituteur adjoint, élève-maître...), le lieu d'exercice (ville, collège, lycée, école normale...), le grade et le régiment, la date de la mort. Outre la liste des tués à l'ennemi et des blessés, les bulletins reproduisent les citations à l'ordre de l'armée ${ }^{12}$.

Les inspecteurs morts au front font l'objet d'une nécrologie spécifique. C'est le cas, par exemple, de M. Barathieu, inspecteur primaire de Montmorillon dans la Vienne, ou de l'inspecteur David en Charente. On y rappelle les étapes de leur carrière et les caractères de l'exercice de leur métier. Barathieu a été fait prisonnier à la bataille de la Somme; il est mort des conséquences de son emprisonnement. Deux pages et demie lui sont consacrées dans le Bulletin départemental de l'instruction primaire de la Vienne ${ }^{13}$.

7. Ibidem, juillet-août-septembre 1914, n² 263, p. 570.

8. Ibid., p. 569.

9. Bulletin départemental de l'instruction primaire (Vienne), $\mathrm{n}^{\circ} 303$, octobre-novembre 1914, p. 202.

10. Bulletin de l'Instruction primaire (Haute-Vienne), $\mathrm{n}^{\circ} 1$, janvier 1915, p. 37.

11. Bulletin de l'Instruction primaire de la Vendée, avril-mai 1915, p. 159.

12. Celui de la Vendée donne aussi la liste des prisonniers et disparus.

13. Numéro 316, juillet-août 1917, p. 239-241. 


\section{Livres d'or au lendemain de la guerre}

Au lendemain de la guerre, toutes ces informations sont rassemblées avec la volonté d'établir de véritables recueils attestant de la participation à la guerre. Plusieurs centaines de livres d'or sont alors publiés en France, car ce n'est ni une spécificité de l'académie de Poitiers, ni une spécificité du monde enseignant. Un projet national de Livre d'or des Français morts pour la France est initié par la loi du 25 octobre 1919 "relative à la commémoration et à la glorification des morts pour la France au cours de la Grande Guerre ». Il s'appuie sur le fichier constitué par le ministère de la Guerre dès 1914 et sur les listes communales ${ }^{14}$. Il est en effet demandé à chaque maire de relever les noms de ceux qui sont tombés pour la patrie. Une première compilation était prévue à l'échelle du département, puis les registres auraient été envoyés à Paris pour être conservés au Panthéon. Mais ce projet n'a pas abouti. Certaines communes ont cependant publié ces listes, constituant des livres d'or de leurs concitoyens morts à la guerre. À côté des listes communales, on édite surtout des livres d'or résultant d'initiatives privées, d'origine corporative. Citons le Livre d'Or des collaborateurs de la Compagnie Générale d'Électricité Morts pour la France 1914$1918^{15}$ ou le Livre d'Or des pharmaciens morts pour la France, 1914-1918 ${ }^{16}$.

Dans le monde de l'Éducation, on inscrit sur un registre les noms d'anciens élèves d'une grande école ou d'un grand lycée, de professeurs et d'instituteurs. Dans l'académie de Poitiers, quelques établissements scolaires en ont publié, comme celui à l'initiative de l'Association Amicale des Anciens Élèves du Collège et de l'École Primaire Supérieure de Châtellerault, qui édite en 1922 un Livre d'Or. Hommage à nos Camarades Morts pour la France $1914-1918^{17}$.

Les listes des maîtres et élèves-maîtres, établies dans le Livre d'or de l'Université publié dans les bulletins départementaux de l'instruction primaire de l'académie, font aussi l'objet de compilations après la guerre, à l'échelle des départements, à l'initiative des Inspections académiques, des amicales des écoles normales d'instituteurs ou des amicales d'instituteurs. C'est probablement dans cet objectif que l'inspecteur d'académie de la Vendée donne en janvier 1920 des instructions complémentaires « en vue de réparer certaines omissions qui se sont produites au cours de la publication des pages du Livre d'Or dans le Bulletin départemental ". Il demande donc des renseignements pour le compléter, en particulier aux « instituteurs ou aux institutrices qui résident actuellement dans la localité de leurs collègues victimes de la guerre ${ }^{18}$ ". Ces compilations peuvent donner lieu à des publications. Du fait de la diversité des initiatives, le contenu

14. Conservées aux Archives Nationales (site de Fontainebleau).

15. s.d., 87 p.

16. Paris, Émile Jammes, 1926, 39 p.

17. Châtellerault, Dupuy \& Bousquet, 1922, 248 p.

18. Bulletin de l'Instruction primaire de la Vendée, Janvier 1920, p. 32. 
de ces livres d'or est très variable, les noms étant accompagnés de notices biographiques plus ou moins développées.

Le livre d'or de l'école normale de La Rochelle présente 64 enseignants qui sont passés par l'école normale de Lagord, soit 32 élèves ou élèvesmaîtres, 28 instituteurs, en poste principalement en Charente-Inférieure ou dans les départements voisins, un professeur, un professeur de l'école normale de La Rochelle, deux anciens professeurs d'école normale, inspecteurs du primaire. Dans la plupart des cas, les fiches biographiques manuscrites (état civil, situation militaire, citations) sont accompagnées de photographies des maîtres, élèves-maîtres et professeurs de l'école normale mobilisés morts pour la France, le plus souvent en uniforme.

Le livre d'or des instituteurs de l'Indre (école normale de Châteauroux) est publié en 1920 par l'Amicale des instituteurs et institutrices publics ${ }^{19}$. Il rappelle que 283 instituteurs de l'Indre ont été mobilisés; 60 sont morts à la guerre, 13 furent prisonniers en Allemagne et 53 ont été blessés. Il donne aussi la liste des instituteurs ayant bénéficié d'une promotion de grade ou ayant reçu une décoration et il reproduit aussi les citations.

Selon une note datée du 21 mai 1933, le livre d'or de l'enseignement primaire de la Charente a été constitué par M. Besson, secrétaire de l'inspection académique de la Charente " depuis le $1^{\text {er }}$ octobre 1913 jusqu'à sa mise à la retraite ${ }^{20}$ ". On y trouve la liste des professeurs et instituteurs de la Charente décorés de la Légion d'honneur et la liste des fonctionnaires tués à l'ennemi ou morts des suites de la guerre. Ces listes sont suivies de fiches individuelles avec les états de service militaire effectué avant et pendant la guerre; elles rendent compte de la diversité des formes de mobilisation des personnels. Si la grande majorité part au front, d'autres sont mobilisés pour les services de l'intérieur et restent sur place, comme Pierre Besson, le secrétaire de l'inspection académique, incorporé au $21^{\mathrm{e}}$ régiment d'artillerie stationné à Angoulême. Ce livre d'or, conservé aux archives départementales de Charente, n'a pas fait l'objet d'une publication.

\section{Des livres d'or aux monuments}

C'est à partir des listes publiées dans le Livre d'or de l'Université que s'élaborent des projets de monuments aux morts.

\section{Les premiers projets}

Dès le début de la guerre, on voit naître des initiatives pour « créer un témoignage permanent du dévouement des instituteurs morts pour la Patrie ", dont rend compte la presse professionnelle. Dans un texte écrit le 15 avril 1916, Édouard Petit, inspecteur général de l'Instruction publique,

19. Amicale des instituteurs et institutrices publics, Guerre de 1914-1918, Livre d'or des instituteurs de l'Indre, Issoudun, L. Sery, 1920.

20. Archives départementales de la Charente, 1T, prov 230. 
évoquant la $75^{\mathrm{e}}$ liste des instituteurs tués à l'ennemi qui vient de paraître au Livre d'or de l'Université, liste " particulièrement chargée ", pense que « sur un monument que les éducateurs élèveront à la mémoire de leurs camarades, tous les noms figureront, quand la paix sera revenue ${ }^{21}$ ". Dans un article du Journal des instituteurs du 24 septembre 1916, intitulé " Nos chers morts", Édouard Petit suggère de donner le nom d'un instituteur ou d'un professeur mort à la guerre à une salle de classe ou à un établissement scolaire :

"En outre des Listes, des Livres d'Or, des Plaques commémoratives, la dédicace du nom serait pour l'Enfance et l'adolescence comme une leçon d'héroïsme permanente et profonde. Et aux jours de cérémonie, de grande solennité, à l'appel du nom glorieux, un élève désigné par ses condisciples répondrait [...] : "Présent" 22 !"

En octobre 1916, un certain nombre d'écoles ont déjà répondu à ce vœu $^{23}$. À Paris, le conseil municipal se propose d'examiner les propositions ou pétitions relatives à l'érection de monuments commémoratifs.

Dès 1916, des amicales pédagogiques proposent l'érection à leurs frais d'une "plaque du souvenir " sur laquelle on graverait les glorieux noms et qui serait placée soit à la préfecture, soit au siège de l'inspection académique, soit à l'école normale des instituteurs ${ }^{24}$. On suggère aussi de rappeler la mémoire des disparus par une inscription, une pancarte, un tableau, un portrait, " placés dans le vestibule de l'école dont les maîtres ont donné leur vie pour la France ", ou mieux encore dans la salle de classe, "au-dessus de la chaire, face aux élèves "; la photographie du maître sera accompagnée d'une inscription rappelant la date et les circonstances de sa mort, la citation, les décorations, " ce qui perpétuera le mieux, dans l'esprit des générations d'élèves qui se succéderont en ce lieu, l'hommage auquel a droit la mémoire de l'héroïque instituteur ${ }^{25}$ ". Une circulaire de l'inspecteur d'académie de la Seine inférieure, adressée aux inspecteurs primaires, va dans ce sens.

Les textes d'Édouard Petit parus dans la presse professionnelle sont rassemblés dans un ouvrage publié en 1917 sous le titre De l'École à la Nation pendant la guerre ${ }^{26}$. Ces textes, écrits et publiés en pleine guerre, sont d'abord le témoignage des actes de l'École pendant la guerre : les œuvres, les quêtes, les grandes journées de secours. Ils traitent de l'enseignement de la guerre à l'École, du devoir qui a conduit l'instituteur à l'armée, de son rôle et son exemple au combat, et surtout de l'hommage au maître mort :

"L'École [...] a montré d'exemple quel rôle elle pouvait jouer dans la Défense matérielle et morale de la Nation; [... L L'École a donné, au front de

21. De l'École à la Nation pendant la guerre par PETrT, Édouard, inspecteur général de l'Instruction publique, président de l'Union nationale des Mutualités scolaires, préface de Léon Bourgeors, Paris, Alcan, 1917, p. 3.

22. Repris par Édouard Petit, De l'École..., op. cit., p. 13 à 16.

23. Journal des instituteurs, dimanche 29 octobre 1916, p. 51-52.

24. Ibidem.

25. Ibid.

26. Petit, Édouard, De l'École..., op. cit. 
bataille et au front de dévouement, la mesure de ce qu'on pouvait attendre de sa doctrine et de son action. [...] l'École a eu vraiment l'esprit de guerre et s'est efforcée de le maintenir dans la Nation ${ }^{27}$."

Car si la guerre a durement éprouvé l'École, elle l'a aussi glorifiée, comme l'écrit le ministre Léon Bourgeois dans la préface de ce livre, où il exprime le souhait qu'un livre d'or soit, dans chaque école, créé à la mémoire des maîtres.

\section{Pour un monument national aux instituteurs morts pour la France}

C'est au cours de la guerre que l'idée se dessine que les maîtres morts au front doivent recevoir un hommage national avec l'édification d'un monument. Lors d'une réunion tenue le 20 février 1916 à Chambéry, les autorités municipales lancent l'idée d'élever, après la guerre, un monument à la gloire des instituteurs. Un comité d'honneur est constitué, composé des grandes personnalités laïques " qui sont les conseils, les amis et les soutiens de l'école républicaine. " Paul Painlevé, Émile Combes, Maurice Barrès, ou Ferdinand Buisson, comptent parmi les membres du comité national.

Dès le lendemain de la victoire, le Comité local d'action, présidé par le maire de Chambéry sous l'égide du Comité national, invite toutes les municipalités françaises à prendre part à cette manifestation en l'honneur des maîtres laïques, " modestes ouvriers de la Démocratie, qui ont conduit à la victoire les élèves auxquels ils ont appris l'amour de la Liberté28 „. C'est sous la forme d'un appel à souscription en vue d'élever un "Monument aux instituteurs morts pour la patrie " que le Comité demande, en janvier 1919, à toutes les villes d'apporter leur pierre à " cet édifice cimenté par le sang des instituteurs de tous les coins de France ». Le monument doit être élevé devant l'École normale supérieure de Saint-Cloud, foyer de formation des futurs maîtres ${ }^{29}$.

Une circulaire ministérielle du 15 juin 1920 organise une collecte dans toutes les écoles primaires de France ${ }^{30}$. Les fonds recueillis sont à adresser au trésorier à la préfecture de Chambéry. L'inspection académique de la Vendée annonce qu'elle se charge de recueillir et d'envoyer les fonds à Chambéry ${ }^{31}$. En 1929, le comité du monument affirme que le monument aux instituteurs morts pour la France de l'École normale supérieure de SaintCloud est terminé, mais qu'il n'a plus de moyens pour son installation et son inauguration; il demande au ministre l'autorisation de faire circuler des listes de souscription dans les écoles primaires ${ }^{32}$.

27. Avant-propos, p. v.

28. Cité dans le Manuel général de l'Instruction publique du 11 janvier 1919, p. 196.

29. Ibidem, Appel à souscription adressé aux municipalités.

30. Bulletin de l'Instruction primaire de la Vendée, juin-juillet 1920.

31. Ibidem.

32. Arch. nat., $\mathrm{F}^{21} 4855$, dossier 68. 
Ce n'est pas un monument dédié aux instituteurs morts pour la France qui est inauguré le $1^{\mathrm{er}}$ novembre 1920 à Saint-Cloud, mais un monument élevé à la mémoire des élèves de Saint-Cloud morts à l'ennemi ${ }^{33}$. Il s'appuie sur un livre d'or des élèves de l'École normale supérieure d'enseignement primaire de Saint-Cloud, publié en $1921^{34}$. D'ailleurs, le monument aux morts, comme le livre d'or, sont financés par l'Amicale : en totalité pour le monument et en partie pour le livre d'or.

Il n'y a donc pas de monument national spécifique pour les instituteurs. Ils font cependant l'objet d'un hommage collectif sur une plaque de marbre " à la mémoire des maîtres morts pendant la guerre 1914-1918 ", à l'initiative de la Fédération des Anciens Combattants de l'Enseignement Public, posée dans le hall d'entrée du musée pédagogique à Paris ${ }^{35}$ et inaugurée le 22 décembre $1934^{36}$. On peut y lire ces chiffres :

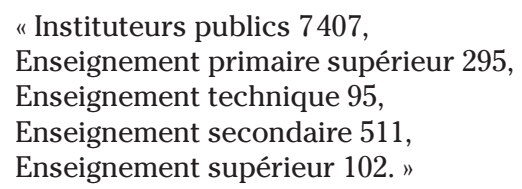

Les monuments dédiés aux instituteurs morts pour la France sont donc locaux, édifiés dans les départements, dans l'enceinte des écoles normales.

\section{Les médailles du ministère de l'Instruction publique}

Les médailles sont une première forme de matérialisation de l'hommage rendu aux enseignants. Dès 1918, le ministère de l'Instruction publique a fait frapper des médailles individuelles spécifiques aux maîtres morts. Réalisées par Victor Prouvé, artiste rattaché au mouvement de l'Art nouveau (École de Nancy), ce ne sont pas des décorations, mais des médailles en bronze, de $6,8 \mathrm{~cm}$ de diamètre ${ }^{37}$. Sur l'avers, on voit un enfant qui dépose une couronne sur un casque posé sur un livre ouvert sur un autel portant l'inscription "Aux maîtres morts pour la France ". Derrière, l'allégorie de la République tient une torche allumée d'une main et pose l'autre main sur l'épaule d'un jeune maître qui vient se recueillir devant l'autel. La scène est cernée d'une inscription : "Instruction publique ". Le revers offre un espace vide entouré de feuilles de chêne et de laurier, destiné à recevoir la gravure du nom d'un mort. Commandées à la Monnaie de Paris par le ministère de l'Instruction publique et des Beaux-Arts, elles sont destinées

33. Le monument aux morts de la Première Guerre mondiale de l'École normale supérieure de Saint-Cloud est installé depuis 2008 dans le hall de l'ENS de Lyon, site Descartes.

34. 1914-1918: Livre d'or de l'École Normale Supérieure d'enseignement primaire de SaintCloud, Nancy, Berger-Levrault, 1921.

35. Aujourd'hui locaux du Institut national d'études du travail et de l'orientation professionnelle, 41 rue Gay-Lussac.

36. L'Université Combattante, $\mathrm{n}^{\circ} 51$, supplément. Bulletin de la Société des professeurs d'histoire et de géographie de l'enseignement public $\mathrm{n}^{\circ}$ 82, janvier 1935, p. 202.

37. Musée de l'École de Nancy, nº d'inventaire 613. 
" à perpétuer dans chaque établissement le souvenir des maîtres morts pour la France ${ }^{38}$ ". La circulaire du 17 février 1920 suggère qu'il faut ajouter à l'hommage du bronze " quelque chose de plus intime, quelque chose où le souvenir du maître apparaisse plus immédiat ", comme un portrait ${ }^{39}$.

Le recteur de l'académie de Poitiers relaie l'initiative ministérielle en annonçant l'arrivée prochaine de ces médailles dans les écoles de l'académie. Il invite les personnels à organiser des cérémonies au cours desquelles seront remises ces médailles, dans les écoles où ces instituteurs enseignaient avant la guerre. Un programme de la cérémonie " à laquelle prendront part tous les enfants de la commune, garçons et filles " est même proposé par le recteur. Un " chant patriotique " sera exécuté par les élèves des écoles et le maire de la commune ou l'inspecteur primaire y prononcera l'éloge du maître disparu. "Cette médaille devra être placée dans une petite vitrine spéciale suspendue au mur au-dessus de la chaire du maître, et rester toujours exposée dans la classe ${ }^{40}$."

Par ailleurs, le recteur recommande " instamment " aux instituteurs de l'école dont un des maîtres est tombé au champ d'honneur de donner " solennellement " à une des salles de l'école le nom de ce maître et de faire inscrire ce nom au-dessus de la porte d'entrée. Ce sera, dit-il, " le moyen le plus efficace de perpétuer parmi les générations d'élèves qui passeront successivement dans cette salle, le souvenir du maître qui a donné, par sa mort héroïque, l'exemple du courage, de l'abnégation et du dévouement absolu à la Patrie ».

Il est aussi prévu de publier dans le bulletin départemental le compte rendu de toutes les cérémonies qui auront lieu dans le département. Ainsi, la remise de la médaille commémorative de $\mathrm{M}$. Point, élève-maître instituteur à Montaigu et officier est l'occasion d'un hommage départemental aux instituteurs de Vendée morts pour la France ${ }^{41}$.

\section{Monuments aux morts, stèles et plaques}

\section{La mémoire s'inscrit dans l'espace}

La forme la plus visible d'hommage aux maîtres morts pour la patrie est l'érection de monuments dans les écoles normales départementales de l'académie. Ces monuments se présentent sous la forme de plaques portant la liste des instituteurs du département morts à la guerre, de stèles ou de véritables " constructions " associant plusieurs éléments : piédestal, obélisque, statues, et inscriptions.

38. Circulaire du ministre de l'Instruction publique du 17 février 1920, Bulletin départemental de l'Instruction primaire (Charente), n 299, mars 1920, p. 373.

39. Ibidem.

40. Bulletin départemental de l'Instruction primaire (Vienne), ${ }^{\circ}$ 331, janvier-février 1920 , p. $199-201$.

41. Allocution de l'inspecteur d'académie de la Vendée. M. Point élève maître instituteur et officier. (Bulletin de l'Instruction primaire de la Vendée, juillet 1920, p. 83-85). 
Dans le monde de l'Éducation, on relève de nombreuses plaques apposées sur les murs des établissements scolaires (écoles, collèges et lycées), rendant hommage aux instituteurs, professeurs, élèves tués au combat entre 1914 et 1918. C'est le cas par exemple dans la cour du Vieux Collège de Châtellerault, actuelle École d'Arts plastiques, où sont inscrits 131 noms, ou de la plaque commémorative des " anciens élèves du collège de Loudun à leurs camarades morts pour la France " dans l'entrée principale du lycée (aujourd'hui Guy Chauvet) ${ }^{42}$.

Dès le lendemain de l'armistice, les établissements scolaires sont incités par leur hiérarchie à constituer des livres d'or et à faire graver sur des plaques les noms des élèves morts pour la France. Dans une circulaire au personnel enseignant " relative à la mémoire de nos élèves morts pour la France ", datée du 12 novembre 1918, le recteur invite les chefs d'établissements et directeurs d'école à consacrer un après-midi à la commémoration de leurs anciens élèves tués à l'ennemi :

« Devant la plaque de marbre sur laquelle nos établissements, de la plus modeste école à la plus haute faculté, tiendront à honneur de conserver gravés les noms de leurs morts, il faut que, tous les ans, les maîtres rappellent la grande guerre avec ses causes, ses vicissitudes, ses conséquences ${ }^{43} \ldots$ ".

Ce sont les associations professionnelles qui sont à l'origine de l'inscription dans la pierre des noms des maîtres morts pour la France. Le monument à la mémoire des instituteurs charentais morts pour la France est érigé "par les soins pieux de l'amicale " des instituteurs de Charente. Dans la Vienne, l'association amicale des anciens élèves de l'école normale d'instituteurs, "la Normalienne ", finance la réalisation des tableaux d'honneur et de la plaque de marbre qui porte le nom des 65 professeurs, élèves et anciens élèves de l'école morts pour la patrie ${ }^{44}$. C'est aussi pour perpétuer le souvenir des 57 maîtres de l'enseignement primaire ou normaliens morts pour la patrie que l'union amicale des instituteurs et institutrices d'Indreet-Loire, de concert avec l'administration académique, ouvre une souscription, parmi le personnel enseignant seulement, pour la pose d'une plaque commémorative dans la cour d'honneur de l'école normale de Loches ${ }^{45}$.

Quelle que soit leur forme, ces monuments sont tous érigés dans l'enceinte des écoles normales des départements du ressort de l'académie, de préférence dans la cour d'honneur. Ce choix est justifié à Poitiers, par le secrétaire de l'association amicale des anciens élèves de l'école normale

42. Inaugurée le 30 mars 1924 en présence du recteur, La Gazette de Loudun du samedi 5 avril 1924.

43. Bulletin de l'Instruction primaire (Haute-Vienne), n 4, octobre novembre 1918, p. 154.

44. Bulletin trimestriel de l'association amicale des anciens élèves de l'école normale d'instituteurs de Poitiers, ${ }^{\circ}$ 3, juillet-septembre 1919.

45. Département d'Indre-et-Loire, Rapport du préfet et procès-verbaux des séances de délibérations du Conseil général, Deuxième session ordinaire de 1920, Annexe n ${ }^{\circ} 5$ - Rapport de l'inspecteur d'académie sur la situation de l'enseignement primaire public et privé dans le département d'Indre-et-Loire pendant l'année scolaire 1919-1920, p. cXXII. 
d'instituteurs, lors de l'apposition de la plaque commémorative le 8 juillet 1920 :

"Cette plaque évocatrice de nos héros tombés sera dans notre vieux Doyenné comme ces tombes encloses dans le bien de famille qu'une touchante coutume poitevine élève parfois, conserve toujours, près de la maison des défunts. Les promotions futures défileront devant elle. Elle entendra des rires jeunes, des chants joyeux ${ }^{46} \ldots$ ".

Pour conserver la mémoire de la Grande Guerre, plusieurs de ces monuments ont été déplacés au gré des déménagements des écoles normales, devenues IUFM, puis ESPE, et ne sont plus sur le site " historique". Le monument de Limoges érigé dans l'ancienne école normale située au lieu-dit Bellevue, devenue Centre d'hébergement et d'éducation des organismes professionnels et sportifs (CHEOPS 87), est déplacé dans les locaux de l'IUFM (aujourd'hui ESPE) sur le campus, boulevard de Vanteaux. À Angoulême, le monument érigé dans la cour d'honneur de l'école, située route de Bordeaux dans le quartier de Sillac, est déplacé une première fois lors de l'installation de l'école normale rue de Varsovie, puis une seconde fois avec l'installation de l'IUFM rue de Montmoreau dans l'ancienne école normale des institutrices de Charente où il est toujours (aujourd'hui site ESPE de la Charente). Certains sont cependant restés sur les sites d'origine. À Parthenay, le monument érigé dans la cour de l'école normale d'instituteurs des Deux-Sèvres est toujours dans la cour de l'établissement devenu collège du Marchioux. À Poitiers, la plaque commémorative fixée sur les murs de l'hôtel Geoffroy d'Estissac qui abritait l'école normale n'a pas suivi le déménagement des services centraux de l'IUFM de Poitou-Charentes (aujourd'hui ESPE de l'académie de Poitiers) sur le campus. À Loches aussi, la plaque commémorative est restée dans l'ancienne école normale de 1885, devenue collège puis lycée Alfred de Vigny, puis centre de loisirs Maurice Aquilon.

\section{L'Instruction primaire rend hommage à ses morts}

Les monuments sont inaugurés entre octobre 1919 et mars 1924. Le premier est celui d'Angoulême, le dernier celui de Parthenay. Lors de l'inauguration du monument charentais, le directeur de l'enseignement primaire, Paul Lapie, félicite l'enseignement primaire charentais pour l'empressement qu'il a mis à glorifier ses morts ${ }^{47}$.

L'année 1920 est celle qui connaît le plus d'inaugurations. Ceci apparaît relativement précoce, dans la mesure où les listes des morts ne sont pas encore complètes. "Le Livre d'Or de l'Université sera-t-il jamais clos?»

46. L'assemblée générale de " la normalienne " le 8 juillet à l'école normale, Bulletin trimestriel de l'association amicale des anciens élèves de l'école normale d'instituteurs de Poitiers, $\mathrm{n}^{\circ} 3$ juillet-septembre 1920, p. 14.

47. Compte rendu de D. Pignon, Bulletin départemental de l'instruction primaire (Charente), n² 296, décembre 1919, p. 274 et suiv. 
se demande d'ailleurs l'inspecteur d'académie d'Indre-et-Loire dans son rapport pour l'année scolaire 1919-1920; " depuis le mois de juillet 1919, d'autres noms se sont ajoutés à ceux qui figuraient au Livre d'Or de l'Enseignement public d'Indre-et-Loire ${ }^{48}$ ".

Des plaques commémoratives ont été apposées sur les murs des écoles normales de Poitiers, de Châteauroux, de Loches et de La Rochelle. À Angoulême, Parthenay, Limoges et La Roche-sur-Yon, ce sont de véritables monuments. Les noms des morts sont inscrits sur les faces d'imposants obélisques, à l'exception du monument de La Roche-Sur-Yon où les listes sont apposées sur les deux côtés du monument qui encadrent un banc de pierre dont le dosseret porte l'inscription " 1914-1918 ». Une sculpture allégorique est debout sur la face avant du monument à Parthenay et à Angoulême et au centre du monument à La Roche-sur-Yon. Dans les trois cas, il s'agit d'une figure féminine qui personnifie l'École. À Angoulême, sa main droite est posée sur l'épaule d'un écolier à qui elle montre de la main gauche l'inscription "Souviens-toi ». À Parthenay, la jeune femme en pleurs, pieds nus, appuie le front et la main droite sur la face principale du monument. À La Roche-sur-Yon, c'est la statue d'une femme en deuil (voilée) qui tient dans la main gauche un livre qu'elle serre contre sa poitrine. S'ajoutent à ces statues divers symboles et allégories : un bouclier et une épée contre la stèle du monument de Parthenay, qui est surmonté d'un coq, des couronnes de feuilles de chêne et de laurier à La Roche-sur-Yon et Parthenay, une palme et des épées à Angoulême. Le monument de Parthenay comporte aussi sur sa face droite un médaillon avec le profil de l'allégorie de la République en Cérès, avec en exergue "République Française, 1870 ", et sur la face opposée, la croix de guerre avec, dans le médaillon, un autre profil de la République avec bonnet phrygien ${ }^{49}$.

Les commanditaires ont fait appel à des sculpteurs locaux. Celui d'Angoulême est le fruit de la collaboration du sculpteur Peyronnet et de l'architecte Baleix, artistes d'importance en Charente dans l'entre-deux guerres. Celui de Parthenay est conçu par Charles Sabouraud, ancien élève de l'école normale.

Dans le département de la Vendée, un monument spécifique est inauguré aux Essarts le 13 mars 1921. Il honore les huit membres de la Mutualité scolaire morts pour la France au cours de la Grande Guerre, et a été érigé à l'initiative de la société pour perpétuer leur mémoire ${ }^{50}$.

Les cérémonies d'inauguration de ces monuments constituent des temps forts de la Mémoire. À Poitiers, le 12 octobre 1919, une grande cérémonie honore la mémoire des normaliens et instituteurs de la Vienne morts

48. Département d'Indre-et-Loire, Rapport du préfet..., op. cit., p. cxxI.

49. Pon-Willemsen, Charlotte, Les allégories de la République sur les monuments aux morts en Poitou-Charentes, Parcours du Patrimoine, ${ }^{\circ}{ }^{\circ}$ 342. Geste éditions, 2008. p. 1, 23, 29,50 et 53 .

50. On exhorte à conserver pieusement le souvenir de leur exemple (Bulletin de l'Instruction primaire de la Vendée, mars-avril 1921, p. 82). 
pour la France pendant la guerre $1914-1918^{51}$. Elle est organisée par l'association des anciens élèves " la normalienne ", l'amicale et le syndicat des instituteurs de la Vienne, et regroupe plus de 800 personnes, personnels enseignants et familles des instituteurs morts au champ d'honneur, et en présence du préfet, du général commandant la subdivision de Poitiers, et du recteur Léon Pineau. À Angoulême, le 31 octobre 1919, le monument est inauguré en présence de nombreuses personnalités, dont le directeur de l'enseignement primaire Paul Lapie, qui représente le ministre, et des " instituteurs et institutrices de la Charente, venus de tous les coins du département pour donner, à leurs vaillants anciens collègues, un témoignage d'admiration reconnaissante ${ }^{52}$ ".

Dans certains départements, l'hommage aux maîtres morts pour la France est encore rendu de nos jours avec un dépôt de gerbe à l'occasion des cérémonies du 11 novembre. Ainsi fait-on chaque année à Limoges, devant le monument qui est dans la cour de l'ESPE, au 209 boulevard de Vanteaux.

\section{Pourquoi cet attachement à souligner le sacrifice des instituteurs?}

\section{Reconnaître l'implication du corps enseignant dans la victoire}

Il s'agit de d'abord de marquer la reconnaissance vis-à-vis des mobilisés et des morts pour la France, reconnaissance due à tous les Français, quelle que soit leur origine sociale ou professionnelle. Dès 1914, dans la première page du Livre d'or de l'Université, l'inspecteur d'académie de la Haute Vienne dit que

" c'est grâce à eux [...] que sera réalisé le beau songe que nous rêvions depuis si longtemps sans oser le dire, le rêve d'une paix enfin sûre, de la force brutale enfin humiliée; c'est grâce à eux qu'un grand souffle de fraternité humaine balayera les lourds nuages, montés d'au-delà des Vosges, qui si longtemps ont éteint le ciel $^{53}$ ".

Il s'agit aussi de mettre en avant l'implication particulière du corps enseignant dans l'effort de guerre, implication que traduisent les chiffres des instituteurs mobilisés et des pertes subies. En janvier 1921, interpellé par un député, le ministre de l'Instruction publique donne une statistique de la participation des instituteurs à la guerre : 34480 ont été mobilisés, dont 28309 dans les formations combattantes, 7407 sont " tombés au champ d'honneur ", 9624 ont été blessés et 11976 ont été cités ${ }^{54}$. Ces chiffres sont

51. Bulletin départemental de l'instruction primaire (Vienne), n 329, juillet-octobre 1919, p. 150 .

52. Compte-rendu de D. Pignon, directeur de l'école annexe, dans le Bulletin Départemental d'Instruction primaire de la Charente, $\mathrm{n}^{\circ} 296$, décembre 1919, p. 274.

53. L'inspecteur d'académie, J. Crévelier, Bulletin de l'instruction primaire (HauteVienne), n 1 , Janvier 1915 p. 39.

54. Revue générale d'administration, vol. 44, janvier-février 1921, p. 374. 
repris dans le journal officiel du 7 avril $1923^{55} .82 \%$ des instituteurs mobilisés l'ont donc été dans les formations combattantes, d'où l'importance des pertes, qui représentent $26 \%$ de tués et $34 \%$ de blessés. Si l'on y ajoute les élèves-maîtres, non comptabilisés dans cette statistique, on perçoit que, non seulement le tribut payé à la guerre a été lourd, plus lourd que dans d'autres catégories socio-professionnelles, mais aussi que la participation tant quantitative que qualitative des maîtres à la guerre a été remarquable. À l'échelle locale, cette participation est soulignée notamment par l'Inspection académique de la Charente qui édite, à l'occasion de la fête du cinquantenaire de l'école laïque en juin 1931, une grande affiche intitulée "L'École et la guerre en Charente " qui fait une présentation comptable du livre d'or de l'enseignement primaire de la Charente, chiffres des personnels mobilisés, cités, morts et blessés ${ }^{56}$.

\section{Des instituteurs soupçonnés de défaitisme}

Lors de la réunion tenue le 20 février 1916 à Chambéry, les promoteurs du comité fondé pour élever, après la guerre, un monument à la gloire des instituteurs, expliquent leurs raisons : "Cette initiative [...] partira de Chambéry afin que le nom de notre ville ne demeurât pas plus longtemps comme un blâme attaché à la grande famille des instituteurs ${ }^{57}$. " Il s'agit d'une référence explicite au congrès de Fédération Nationale des Syndicats d'Instituteurs, tenu dans cette même ville du 15 au 17 août 1912. On y vote " le sou du soldat ", caisse de solidarité destinée à aider les appelés et permettre de maintenir un lien entre les syndicats et leurs adhérents pendant qu'ils effectuent leur service militaire. Le gouvernement considère cette initiative comme un moyen de propagande antimilitariste, d'autant que le vote se serait accompagné de "déclarations antipatriotiques ${ }^{58}$ ". Les syndicats d'instituteurs sont mis en demeure de "se dissoudre ". Les instituteurs répliquent à l'offensive du gouvernement par un manifeste où, après avoir affirmé leur attachement à la patrie, ils se déclarent " résolument pacifistes" :

"Nous croyons tout proche le moment où les conflits internationaux se régleront sans effusion de sang, de par la volonté souveraine des peuples intéressés. Et nous ne saurions trop protester contre les excitations chauvines et les manœuvres de politiciens et financiers qui risquent à chaque instant de provoquer une conflagration générale ${ }^{59}$."

55. Journal officiel, 7 avril 1923, p. 1732. C'est aussi le chiffre qui est donné sur la plaque commémorative du Musée pédagogique.

56. Affiche "Cinquantenaire de l'école laïque, programme de la fête ", Arch. dép. de la Charente, 1TProv 230.

57. Manuel général de l'Instruction primaire, n 16, 11 janvier 1919, p. 196.

58. SÉVERAC, Jean-Baptiste, "Le gouvernement contre les Instituteurs syndicalistes ", Le Mouvement Socialiste, III ${ }^{\mathrm{e}}$ série, juin-décembre 1912, p. 246.

59. Cité par DEFRASNE, Jean, Le pacifisme, Paris, PUF, 1983, p. 74. 
La FNSI est dissoute après le congrès de Chambéry. Du fait de cette prise de position, "l'identification instituteurs-antimilitarisme sera véhiculée par la suite de façon abusive " dit l'historien Jacques Girault ${ }^{60}$. En effet, l'opposition à la guerre n'a été qu'un phénomène marginal dans le corps enseignant ${ }^{61}$, même si elle a existé, y compris dans l'académie de Poitiers, notamment avec les époux Mayoux (François et Marie), instituteurs de Charente syndiqués à la CGT, révoqués et condamnés à une peine d'emprisonnement pour propagande pacifiste ${ }^{62}$.

Dénonciateur de l'antimilitarisme, Émile Driant s'émeut des « déclarations antimilitaristes et antipatriotiques du congrès de Chambéry ". Pour combattre des idées qu'il considère comme néfastes, il décrit en 1912 une guerre imaginaire sous la forme d'un roman, Robinsons souterrains, publié sous le pseudonyme de Capitaine Danrit ${ }^{63}$, qui est une attaque en règle contre les pacifistes et dans lequel il fait d'un instituteur un congressiste de Chambéry, un " mauvais soldat " et un «mauvais Français ".

\section{Effacer le congrès de Chambéry}

Dès 1916, des personnalités du monde politique et littéraire, reconnaissent l'engagement des instituteurs, qui fait contraste avec les positions adoptées à Chambéry. Louis Barthou, journaliste et député, plusieurs fois ministre et président du Conseil en 1913, constate dans Les Annales, le 8 octobre 1916, que "nos instituteurs se sont bien battus ". Si la censure lui interdit de donner le nombre des membres de l'enseignement qui sont tombés ou qui ont été blessés en service de guerre, il peut affirmer que « la liste des instituteurs est significative et émouvante ", à commencer par le premier mort français en 1914, Jules André Peugeot, qui était instituteur. Ainsi, quand cette liste sera connue, " elle constituera la plus noble réponse à des détracteurs dont beaucoup déjà se sont honorés en reconnaissant ou en réparant leur erreur ${ }^{64}$ ". Il peut citer le tableau d'honneur, donné trois mois auparavant, qui fait apparaître que l'enseignement public a recueilli 3000 citations diverses, 170 croix de la Légion d'honneur et 180 médailles militaires. " Les membres de l'enseignement primaire ont une large part dans ce glorieux tableau d'honneur et on ne fera jamais trop pour perpétuer leur souvenir. " Barthou reproduit une lettre écrite par un instituteur mobilisé, plusieurs fois décoré et nommé commandant, à sa femme institutrice :

60. GiRAUlT, Jacques, « Histoire du syndicalisme chez les instituteurs jusqu'à la Seconde guerre mondiale ", Histoire de l'Éducation, décembre 1979, n 5, p. 7.

61. GIRAULT, Jacques, Instituteurs, professeurs : une culture syndicale dans la société française (fin $X I X^{e}-X X^{e}$ siècles), Paris, Publications de la Sorbonne, 1996, p. 150-153.

62. Mayoux, Marie et François, Instituteurs pacifistes et syndicalistes (Mémoires de F. Mayoux), introduction de Daniel Guérin, postface de Madeleine Rebérioux, Chamalières, Éditions Canope, 1992, 363 p.

63. Capitaine DANRIT (Lt-Colonel DRIANT, Émile), Robinsons souterrains, Paris, Flammarion, 1913.

64. Annales, 8 octobre 1916, p. 572. Texte repris dans le Journal des instituteurs, dimanche 29 octobre 1916, p. 51-52. 
"Si je rentre dans l'enseignement après la guerre, comme je l'espère, je serai cette fois qualifié pour faire des leçons sur la patrie et je ne serai sans doute plus appelé dangereux antipatriote et anarchiste du congrès de Chambéry."

Barthou revient sur les égarements du congrès de Chambéry, qui procédaient " d'un humanitarisme sentimental et d'un internationalisme crédule dont l'agression a rudement secoué les illusions ". Quelques-uns des congressistes, "revenus de leur rêve, ont pris le fusil et sont tombés face à l'ennemi "; leur mort " a tout réparé et tout effacé. Entre les instituteurs de France et la France, il n'y a plus de malentendu ".

Raymond Thamin, philosophe et universitaire, est encore plus explicite. Dans un article de la Revue des deux Mondes $^{65}$, il fait référence à la conduite des normaliens en 1870 :

"À l'entrée même de l'école normale, notre génération saluait pieusement la plaque commémorative du normalien mort pour la patrie. Demain, le péristyle de la vieille maison sera trop étroit pour les commémorations dues [...]; cette présente guerre était la première où, en masse, professeurs et instituteurs étaient appelés à l'honneur de se battre..."

Le recteur Thamin renvoie les lecteurs au bulletin de l'instruction publique et à toutes les revues d'enseignement " qui s'ouvrent, depuis bientôt deux ans, par la liste glorieuse, certains jours effroyablement longue, des morts pour la patrie ${ }^{66}$ ". Les " vertus militaires " des instituteurs engagés sur le front ont causé à ceux qui les connaissaient mal, et les " jugeait d'après des manifestations tapageuses qui n'engageaient que ceux qui s'y livraient ", une " agréable surprise ". Peut-être même, ajoute-t-il, ont-ils mis un point d'honneur à se montrer plus braves parce qu'instituteurs. "Les chefs de ceux qu'on appelait les syndicalistes sont morts les premiers ", à commencer par Cren, " qui présida le Congrès autrefois fameux (mais combien cela nous paraît lointain!) de Chambéry ${ }^{67}$ ". Paul Lapie, directeur de l'enseignement scolaire, constate " avec satisfaction que, parmi ceux qui allaient partir, les "pacifistes" les plus notoires [...] n'étaient pas les moins ardents ${ }^{68}$ ".

C'est en feuilletant le Bulletin de l'instruction primaire du département de l'Indre, "dans toutes ses parties consacrées aux instituteurs de l'Indre morts pour la patrie, aux instituteurs blessés, aux instituteurs cités et décorés, aux œuvres de guerre du département ", que Maurice Barrès reconnaît que, s'il a été avant la guerre " en bataille avec un certain nombre d'instituteurs ", cette position est désormais bien loin ${ }^{69}$.

65. "L'Université de France et la guerre ", Revue des deux Mondes, tome 34, juilletaoût 1916, p. 294-324 et 586-618.

66. Ibidem, p. 300.

67. Ibid., p. 302.

68. LAPIE, Paul, L'instituteur et la guerre, articles documents publiés dans la Revue pédagogique, repris en volume, Paris, Didier, coll. " La guerre et l'École ", 1915, p. 58.

69. BARRÈs, Maurice, L'âme française et la guerre, tome 9, Pendant la bataille de Verdun, 1919, Émile-Paul frères, Paris, p. 40. 
Driant lui-même opère une refonte de son roman Robinsons souterrains, republié sous le titre La guerre souterraine en 1915, avant que son auteur ne soit tué à Verdun en février 1916. Il reconnaît que les instituteurs, qu'il a vu servir et mourir à ses côtés "se sont ressaisis ", qu'ils " ont compris que, pour effacer le souvenir d'un passé trop récent, ils devaient prêcher d'exemple et la liste de leurs morts, le Tableau d'Honneur de leurs citations ont, depuis les premières batailles, fait oublier les déclarations de leurs congrès ". Il a donc en 1915 supprimé dans son livre « le personnage odieux de l'intellectuel antipatriote ", en même temps que, fidèle à l'Union sacrée, il a "rayé de sa mémoire les douloureuses manifestations de certains instituteurs d'avant guerre ${ }^{70} "$.

\section{Servir d'exemple aux futures générations de maîtres}

L'attitude des instituteurs dans la guerre a donc une valeur exemplaire. Le choix des écoles normales pour l'érection des monuments est significatif de cette leçon. Le monument national qui doit être érigé à Saint-Cloud "montrera aux jeunes ce que furent leurs aînés ${ }^{71}$ ".

Devant le monument dédié aux instituteurs de Charente morts pour la patrie, le directeur de l'enseignement scolaire montre comment l'héroïsme des instituteurs est inspiré par l'enseignement moral de l'École, car cet enseignement " lui ordonne de se subordonner et de sacrifier à la collectivité ". Il engage les élèves-maîtres à venir pieusement méditer devant le monument élevé dans la cour de l'école en souvenir de leurs anciens pour " accomplir en toute circonstance leur devoir envers leur pays ${ }^{72}$ ".

Quand il inaugure le monument de l'école normale de Limoges le 27 juin 1920, le recteur Léon Pineau fait le vœu que chaque année les élèvesmaîtres s'y recueilleront, qu'ils épelleront les noms, et qu'ils accompliront leur " devoir de guerre ", qui est le thème de son discours, fidèles au mot d'ordre transmis par les anciens ${ }^{73}$.

Cette exemplarité, ce modèle à suivre pour les jeunes générations de maîtres, est un leitmotiv des discours d'hommage aux morts de l'École que le recteur Pineau a rassemblés dans un ouvrage. À Loches, le 16 novembre 1920, s'adressant à nouveau aux " élèves-maîtres, éducateurs de demain ", il leur rappelle ce qui doit les inspirer :

70. Capitaine DANRIT (Lt-Colonel DRIANT, Émile), La Guerre souterraine (Robinsons souterrains), Paris, Marpon et Flammarion, 1915, préface.

71. Appel à souscription adressé aux municipalités, Manuel général de l'Instruction publique, 11 janvier 1919, p. 196.

72. "Chronique de l'enseignement primaire en France ", Revue pédagogique, nouvelle série, tome $76, \mathrm{n}^{\circ} 1$, janvier 1920 , p. 67.

73. Le texte du discours est intitulé « Le patriotisme à l'école et le rôle social de l'Instituteur ". Bulletin départemental de l'Instruction primaire (Vienne), $\mathrm{n}^{\circ} 336$, juillet-septembre 1920, p. 397. 
"Pas plus que vos aînés, vous ne faillirez à la tâche. Sans doute votre action dans la paix sera-t-elle moins glorieuse que ne fut la leur dans la guerre : vous la ferez grande aussi et noblement féconde en comprenant, comme ils l'ont compris, et en pratiquant, que c'est par le dévouement à son pays que l'on sert mieux l'humanité ${ }^{74}$."

L'étude des modalités et du contexte de mise en place de l'hommage aux instituteurs de l'académie de Poitiers morts à la guerre met en évidence la précocité et la diversité des manifestations mémorielles initiées par l'École publique. Elle traduit une volonté de faire connaître l'adhésion des instituteurs à l'Union sacrée, ce qui a pour conséquence de conforter le rôle de l'instituteur et par-delà celui de l'École dans la nation. Cette forme d'implication de l'École dans la guerre qui se traduit par la mobilisation de ses maîtres sur le front, est à compléter par la mobilisation de l'École à l'arrière par les " œuvres de guerre ", mobilisation à laquelle il est fait aussi allusion dans les discours.

Les initiatives mémorielles prises à l'échelle locale dans l'académie de Poitiers attestent de la systématisation de l'hommage aux " maîtres morts pour la France " dans toutes les académies, ce qui lui donne une dimension nationale qui confirme la spécificité de cette mémoire.

74. PinEAU, Léon, Pour vaincre dans la paix, Paris, Bloud et Gay, 1923, p. 201. 
Résumé

Cet article s'intéresse aux origines et aux formes de l'hommage rendu aux instituteurs tués au combat pendant la Première guerre mondiale. Cet hommage s'organise précocement, avant même la fin de la guerre et connaît son plein développement dans les premières années d'après-guerre. Il est orchestré localement, principalement par le rectorat et les inspections académiques, dont les initiatives sont relayées par les associations professionnelles. L'académie de Poitiers qui comprend alors huit départements est représentative de cette mise en mémoire, qui voit se multiplier, dans toute la France après la guerre, les livres d'or et les monuments dédiés aux maîtres morts pour la patrie. Érigés dans les écoles normales départementales, les monuments aux instituteurs témoignent d'une volonté de souligner leur sacrifice dans un souci d'exemplarité et de montrer la participation à l'effort de guerre et à la victoire des enseignants du primaire, longtemps soupçonnés de défaitisme.

\section{Abstract}

This article explores the origins and forms of the tribute paid to primary school teachers killed in action during the First world war. This tribute was organised early, even before the war ended and was fully developped in the first years after the war. It was orchestrated by the local education authority, the Rectorat and Inspection académique, whose initiatives were relayed by professional associations. The local education authority of Poitiers, which then comprised eight departments, was representative of the shaping of the remembrance of the war. This was characterised, everywhere in France after the war, by the creation of Golden Books and monuments to the teachers who fought and died for their country. Erected in the institutions where primary teachers were trained, monuments were dedicated to the memory of those killed. This showed a willingness to acknowledge their sacrifice and their exemplary behaviour. It also highlighted the role of primary school teachers, who were long suspected of defeatism, in the war effort and eventual victory. 